\title{
Evaluation and diagnosis of gallstone ileus using point of care ultrasound in the Emergency Department
}

\author{
Steven Van Rees, Kristen Price, Joe Betcher \\ Mercy Health Partners, Muskegon, MI, USA
}

\begin{abstract}
A 75-year-old female presented to the Emergency Department with nausea, feculent emesis and abdominal distension. On previous visit the patient was diagnosed with a $2.2 \times 1.9 \times 1.1 \mathrm{~cm}$ ectopic gallstone in the mid small bowel without evidence of ileus or bowel obstruction and was subsequently discharged home. She returned 2 days later, abdominal plain radiographs revealed mildly dilated small bowel with air-fluid levels and a calcified mass in the right hemipelvis. Bedside ultrasound was performed and an airfilled gallbladder with a fistulous tract was visualized. Dilated loops of bowel were visualized with an abnormal back and forth movement of intestinal contents, consistent with a small bowel obstruction. Patient went on to have an uncomplicated surgical extraction of the gallstone with resolution of her ileus. We would propose using point-of-care ultrasonography with abdominal plain films as an adjunct for expedius bedside evaluation and diagnosis of gallstone ileus in elderly patients.
\end{abstract}

\section{Case Report}

A 75-year-old female presented to the Emergency Department (ED) with nausea, feculent emesis and abdominal distension.

Correspondence: Joe Betcher, Mercy Health Partners, 16298 Highland Dr, Spring Lake, MI 49456, USA.

Tel.: +1.616.403.6908 - Fax: +1.231.727.5223.

E-mail: joebetcher@gmail.com

Key words: Gallstone ileus; ultrasound; cholecysto-enteric fistula.

Contributions: JB, KP, ultrasound images; SVR, care of the patient. SVR, KP, original manuscript drafting, images collection and editing. All authors reviewed and provided edits for the final copy of the manuscript.

Conflict of interest: No one. This work was not supported by any grant. Availability of data and materials.

Ethics approval and consent to participate: The manuscript does not contain any elements that would allow the recognition of the patient.

Received for publication: 18 September 2019.

Accepted for publication: 28 October 2019.

This work is licensed under a Creative Commons Attribution 4.0 License (by-nc 4.0).

(OCopyright: the Author(s), 2020

Licensee PAGEPress, Italy

Emergency Care Journal 2020; 16:8570

doi:10.4081/ecj.2020.8570
Point-of-care ultrasound and abdominal plain films were used to identify and diagnose bowel obstruction due to a cholecystoenteric fistula, also referred to as gallstone ileus. The patient initially presented with nausea and non-bilious emesis and was found to have a $2.2 \times 1.9 \times 1.1 \mathrm{~cm}$ ectopic gallstone in the mid small bowel with evidence of a fistulous tract between the gallbladder and proximal small bowel on non-contrast enhanced computed tomography (CT) of the abdomen and pelvis. The patient did not appear to have an associated ileus or bowel obstruction on initial presentation clinically or radiographically. She was ultimately discharged home with close outpatient follow up with general surgery. She returned to the ED 2 days later with recurrent nausea, feculent emesis, abdominal distension, constipation and obstipation. Bedside ultrasound was performed and an air-filled gallbladder with a fistulous tract to an intestinal loop was visualized. Dilated loops of bowel were visualized with an abnormal back and forth movement of intestinal contents, consistent with dysfunctional peristalsis associated with a small bowel obstruction. Abdominal plain radiographs revealed mildly dilated small bowel with air-fluid levels and a calcified mass in the right hemipelvis. Ultrasonography combined with abdominal plain films was consistent with the patient's clinical picture, a case of gallstone ileus due to a cholecysto-enteric fistula. Her care was expedited due to rapid diagnosis and she did not require repeat CT imaging.

\section{Discussion}

Gallstone ileus is a rare complication of chronic recurrent cholecystitis in which a fistulous tract is created between the biliary and enteric systems resulting in bowel obstruction due to an ectopic gallstone. ${ }^{1}$ Gallstone ileus disproportionately affects elderly females. ${ }^{1-6}$ Diagnosis is difficult in this population due to multiple comorbidities and nonspecific presentations. As a result, diagnosis is often delayed resulting in high morbidity and mortality up to $20-40 \% .^{5,7,8}$ Recurrent episodes of cholecystitis in presence of cholelithiasis causes chronic inflammatory changes which eventually causes the walls to become necrotic resulting in a fistulous tract from the gallbladder into the small intestine. Stones greater than $2 \mathrm{~cm}$ become obstructed causing small bowel obstruction with the most common site being the distal ileum or ileocecal junction. ${ }^{3,4}$ Early diagnosis in the ED is key to preventing morbidity and mortality and thus we would propose considering point-of-care ultrasonography as a mode of rapid and efficient diagnosis of gallstone ileus in conjunction with confirmatory tests such as CT imaging.

While CT remains the gold standard for diagnosis, there are patients who do not tolerate CT imaging in the ED. . $^{5-11}$ Additionally, CT imaging does not reliably identify ectopic gallstones as visualization is dependent on stone composition. .,12,13 $^{-1}$ Ultrasonography has been found to be a useful tool in diagnosing gallstone ileus at bedside when combined with abdominal plain films, with sensitivities as high as $74 \%{ }^{12}$ It is cost effective, 
efficient and without exposure to ionizing radiation or intravenous contrast when compared to CT imaging. A cholecysto-enteric fistula is visualized as hyperechoic areas with acoustic shadowing within the gallbladder or non-visualization of gallbladder due to distended air-filled intestines, bowel obstruction is visualized as an abnormal back and forth movement of intestinal contents within dilated loops of bowel, and gallstones consistently appear as ectopic hyperechoic masses with posterior acoustic shadowing.

We would propose using point-of-care ultrasonography
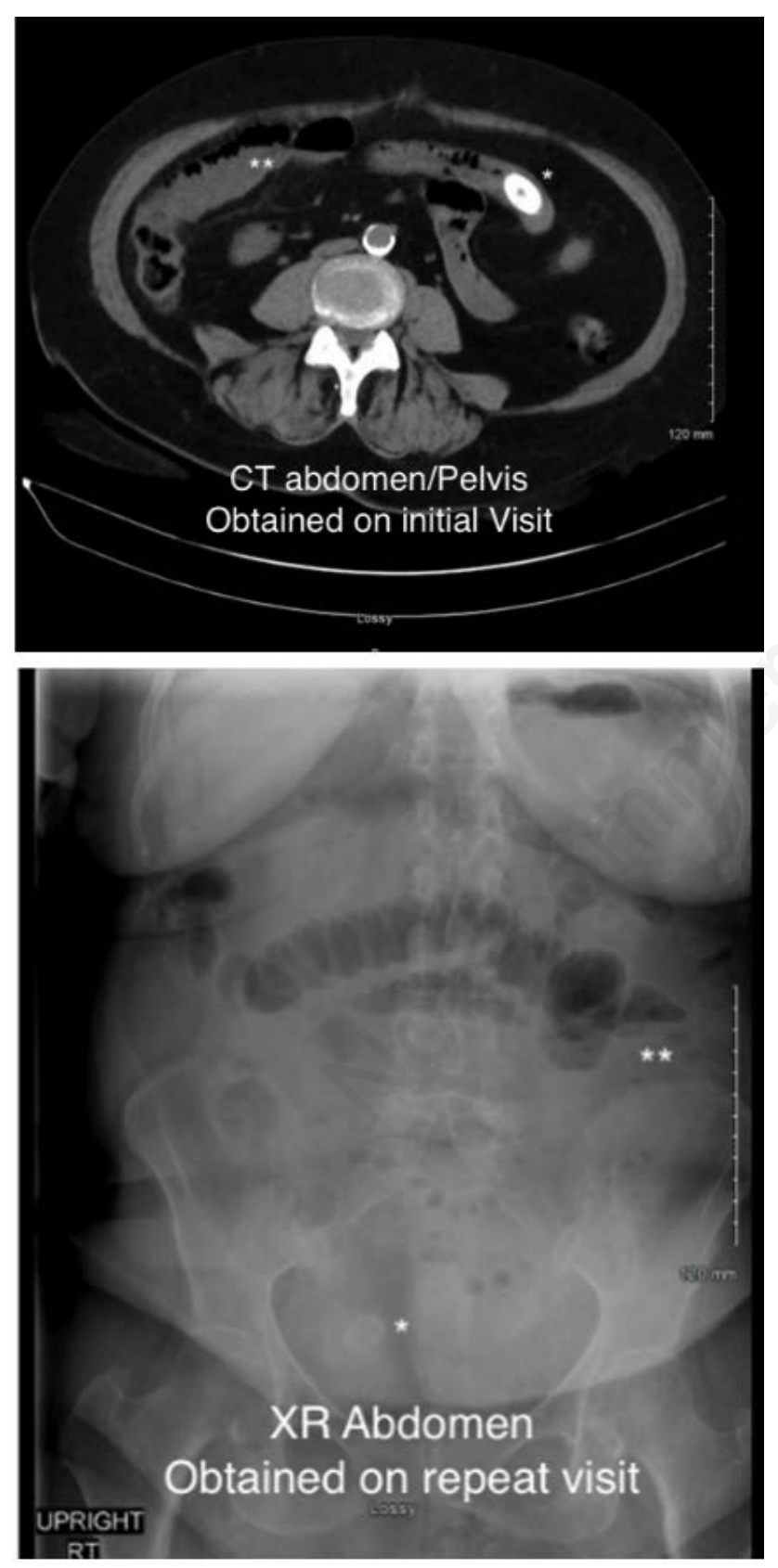

Figure 1. Abdominal computed tomographic (CT) scan and Xray $(\mathrm{XR})$ demonstrating air fluid levels, small bowel dilation and presence of an ectopic gallstone near location of terminal ileum. ${ }^{*}$ Denotes location of ectopic gallstone; ${ }^{* *}$ Denotes presence of air fluid levels.
(POCUS) with abdominal plain films as an early adjunct for evaluation and diagnosis of gallstone ileus in elderly patients to expedite care. CT imaging, with or without contrast, should be obtained to rule out other possible etiologies for bowel obstruction. Her ileus was diagnosed using abdominal plain films which visualized a small bowel obstruction and an ectopic stone. Bedside ultrasonography was used to visualize aerobilia as well as small bowel obstruction confirming her final diagnosis of gallstone ileus. She was expeditiously admitted, went on to have a diagnostic laparoscopy followed by mini laparotomy with enterotomy and gallstone removal. The patient fortunately had an uncomplicated postoperative recovery and was discharged on postoperative day three.

\section{Conclusions}

Point-of-care ultrasonography is a valuable diagnostic tool in the evaluation and diagnosis of gallstone ileus in the emergency department. It increases the sensitivity to diagnose gallstone ileus when combined with abdominal radiographs. It has particular benefits over CT including efficiency, cost, radiation and is without intravenous contrast. POCUS should be considered a useful adjunct to diagnosis of gallstone ileus to expedite diagnosis and treatment. CT imaging should remain the gold standard to evaluate and rule out other etiologies of small bowel obstruction.

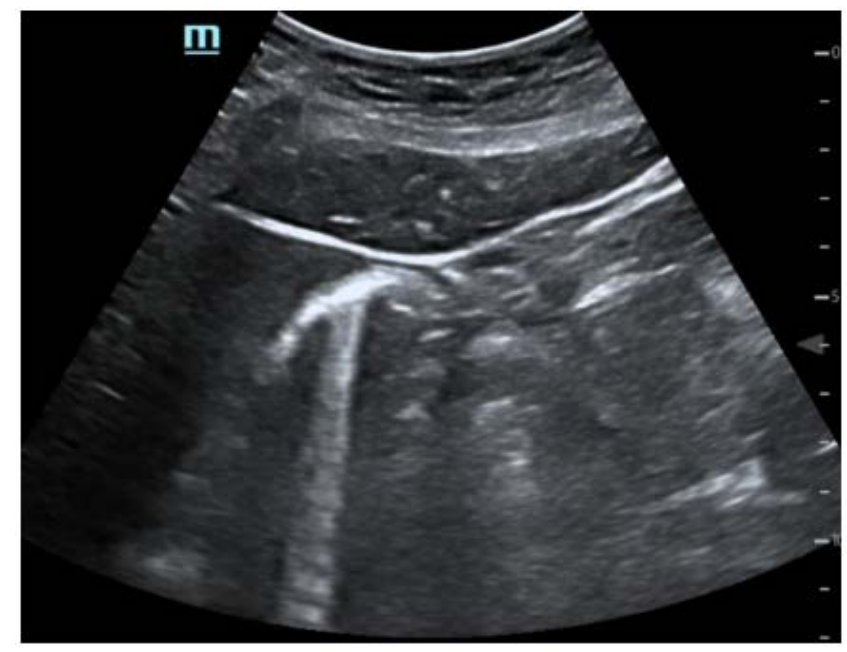

Figure 2. Ultrasonography still image showing gas within gallbladder concerning for a fistula tract between gallbladder and small bowel. 

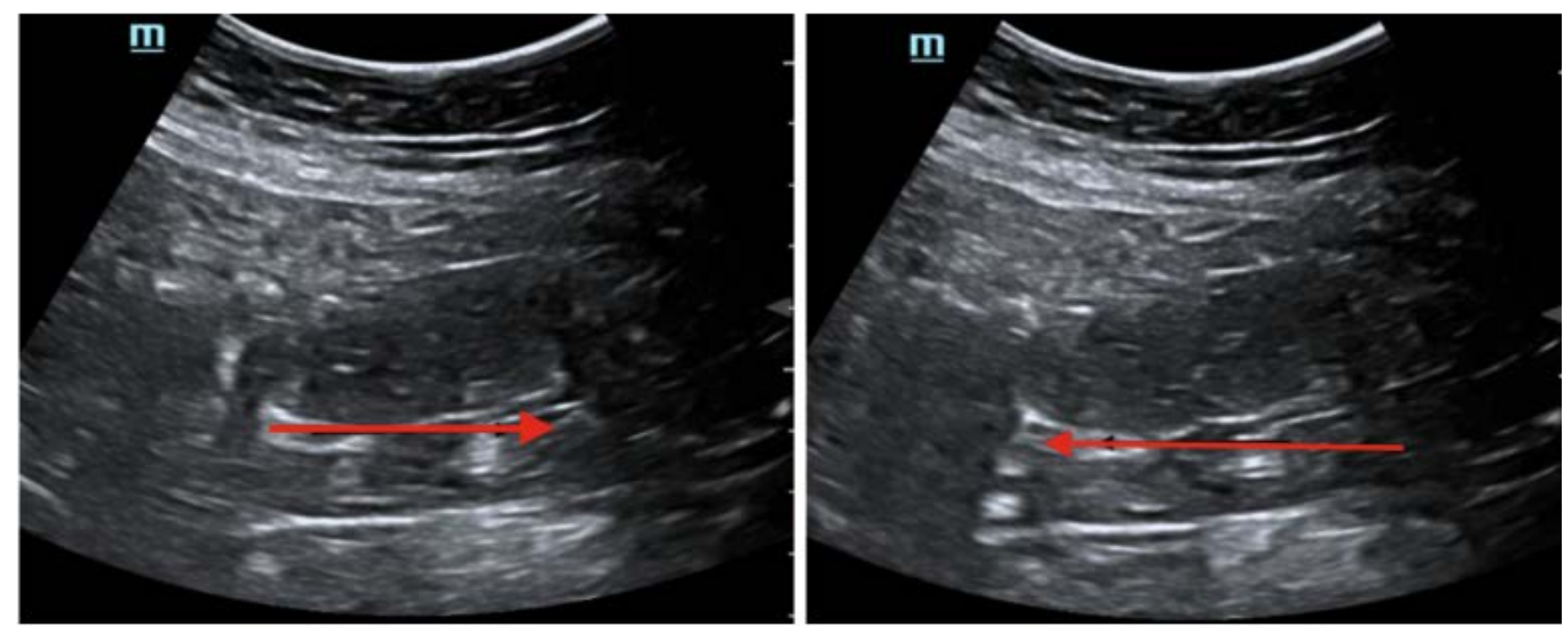

Figure 3. Ultrasonography small bowel contents circulating in a back and forth pattern concerning for small bowel obstruction. Arrow denotes movement of intestinal fluid in one direction.

\section{References}

1. Artioli G, Muri M, Praticò F. Gallstone ileus: literature review. Acta Biomed 2016;87:40-4.

2. Buljevac M, Busic Z, Cabrijan Z. Sonographic diagnosis of gallstone ileus. J Ultrasound Med 2004;23:1395-8.

3. Reisner R, Cohen J. Gallstone ileus: a review of 1001 reported cases. Am Surg 1994;60:441-6.

4. Rodríguez J, Codina A, Gironès J. Gallstone Ileus results of analysis of a series of 40 patients. Gastroenterol Hepatol 2001;24:489-94.

5. Chang L, Chang M, Chang H. Clinical and radiological diagnosis of gallstone ileus: a mini review. Emerg Radiol 2018; 25:189-96.

6. Chuah P, Curtis J, Misra N. Pictorial review: the pearls and pitfalls of the radiological manifestations of gallstone ileus. Abdom Radiol 2017;42:1169-75.

7. Svarholm E, Andren-Sandberg A, Evander A. Diagnosis and treatment of gallstone ileus: report of 83 cases. Acta Chir Scand 1982;148:435-8.

8. Schutte H, Bastias J, Csendes A. Gallstone ileus. Hepatogastroenterology 1992;39:562-5.

9. Rotaru M, Necula A, Caraiani C. Point-of-care ultrasound in management of gallstone ileus - a case report. Med Ultrason 2019;21:197-9.

10. Stagnitti F, Tudisco A, Ceci F, Nicodemi S. Biliodigestive fistulae and gallstone ileus: diagnostic and therapeutic considerations. Our experience. G Chir J Surg 2014;35:235-8.

11. Lassandro F, Gagliardi N, Scuderi M. Gallstone ileus analysis of radiological findings in 27 patients. Eur J Radiol 2004; 50:23-9.

12. Ripollés T, Miguel-Dasit A, Errando J. Gallstone ileus: increased diagnostic sensitivity by combining plain film and ultrasound. Abdom Imaging 2001;26:401-5.

13. Chawla A, Bosco J, Lim T. Imaging of acute cholecystitis and cholecystitis-associated complications in the emergency setting. Singapore Med J 2015;56:438-44. 